\title{
Drug utilization pattern of antihypertensive drugs in chronic kidney disease patients in a tertiary care hospital
}

\author{
Pavitra R Y ${ }^{1}$, Geetha $\mathrm{M}^{1}$, Rajeev Aggarwal ${ }^{2}$, Somashekar H S ${ }^{1}$ \\ ${ }^{I}$ Department of Pharmacology, JJM Medical College, Davangere, Karnataka, India \\ ${ }^{2}$ Department of Nephrology, JJM Medical College, Davangere, Karnataka, India
}

\begin{abstract}
:
Objectives: To analyze utilization of antihypertensive drugs in chronic kidney disease patients in a tertiary care hospital.

Materials and Methods: A descriptive prospective study was conducted in the dialysis unit of Bapuji hospital, Davangere during 6 month period in 2013 with stage III to V of chronic kidney disease patients. Data from case record of all patients was collected, relevant information entered in a preformed proforma and analyzed.

Results: Total of 120 case records were analyzed, $30 \%$ patients were 51-60 years, $27 \%$ patients were 61-70 years and male to female ratio of 74: 26. Out of 120 patients, 54 patients (45\%) were diabetic with hypertension and 66 patients (55\%) were nondiabetic with hypertension. In diabetic patients with hypertension, $66.6 \%$ were two drugs combinations, cilnidipine and torsemide (52\%), amlodipine and furosemide (28\%) metoprolol and cilnidipine (11\%). 30\% with three drug combinations. Nondiabetic patients with hypertension, $55 \%$ had three drugs combinations, Metroprolol, furosemide, prazosin (45\%), Cilnidipine, torsemide, prazosin (33\%) and $45 \%$ two drugs combinations, amlodipine, furosemide (46\%) and metoprolol, torsemide (33\%) were commonly used.

Conclusion: Combination of diuretic and calcium channel blockers (80\%) was commonly used in hypertensive patients with diabetic. Beta blocker because of its known adverse effects in diabetic patients (hypoglycemic unawareness) is getting less commonly used than in nondiabetic. Use of antihypertensive drugs such as selective beta blocker, alpha-blocker, Calcium channel blocker combination (45\%) more commonly used in nondiabetic with hypertension.
\end{abstract}

Keywords: antihypertensive drugs, chronic kidney disease, diabetic

\section{Introduction}

Chronic kidney disease (CKD) is a major public health issue because of rising incidence, poor outcome and expensive treatment, thereby imposing a burden on social and health care system. According to the World Health Organization (WHO) Global Burden of Disease Project, kidney disease contributes to nearly 850,000 deaths per year along with other urinary tract problems ${ }^{[1]}$. CKD is associated various complications and comorbidities. Hypertension is most frequently seen in chronic renal disease and may cause progressive renal damage if uncontrolled. In order to achieve and maintain adequate blood pressure control various antihypertensive drugs combinations were employed.

The study of prescribing patterns is a component of medical audit that monitors and evaluates prescribing practices and recommends necessary modifications to achieve rational drug use ${ }^{[15]}$. Due to limited literatures available in prescribing various drug combinations, advantage and side effects in chronic renal disease patients to the practising clinicians. Hence, our study was planned to analyse current prescribing trends in the management of CKD patients with hypertension and to suggest ways to rationalize drug use, minimise medication error and improve therapeutic outcome.

\section{Materials And Methods}

A descriptive prospective study was conducted in the Nephrology unit of Bapuji hospital attached to JJM Medical College, Davangere during period of 6 months in 2013 with stage III to V of chronic kidney disease. Study was approved by institutional ethical committee and written informed consent was obtained from each patient before enrolment. All patients diagnosed to have chronic renal disease were undergoing treatment in the nephrology unit were included and their prescriptions were analyzed. Children, pregnant and lactating women, surgical conditions like kidney stone, tumors and trauma were excluded. Demographic, clinical data and medication details were collected in a specially designed proforma. Data on utilization of different classes as well as individual drugs were subjected to descriptive analysis. 


\section{Observations And Results}

Total of 120 case records were analyzed, 54 patients (45\%) were diabetic with hypertension and 66 patients $(55 \%)$ were nondiabetic with hypertension. 30\% patients were 51-60 years, $27 \%$ patients were 61-70 years and male to female ratio of 74: 26.

In diabetic patients with hypertension, $66.6 \%$ were prescribed two drugs combinations and $30 \%$ three drugs combinations. Two drugs combinations commonly used were cilnidipine + torsemide (52\%), amlodipine + furosemide $(28 \%)$ and metoprolol + cilnidipine $(11 \%)$. Three drug combinations used were Metoprolol + cilnidipine + prazosin $(62 \%)$ and Metoprolol + torsemide + Prazosin (25\%). In nondiabetic with hypertension, $55 \%$ with three drug combinations, Metroprolol + furosemide + prazosin $(45 \%)$ and Cilnidipine + torsemide + prazosin (33\%) and $45 \%$ had two drugs combinations , amlodipine+ furosemide (46\%), metoprolol+ torsemide $(33 \%)$ were commonly used.

\section{Figures And Tables}

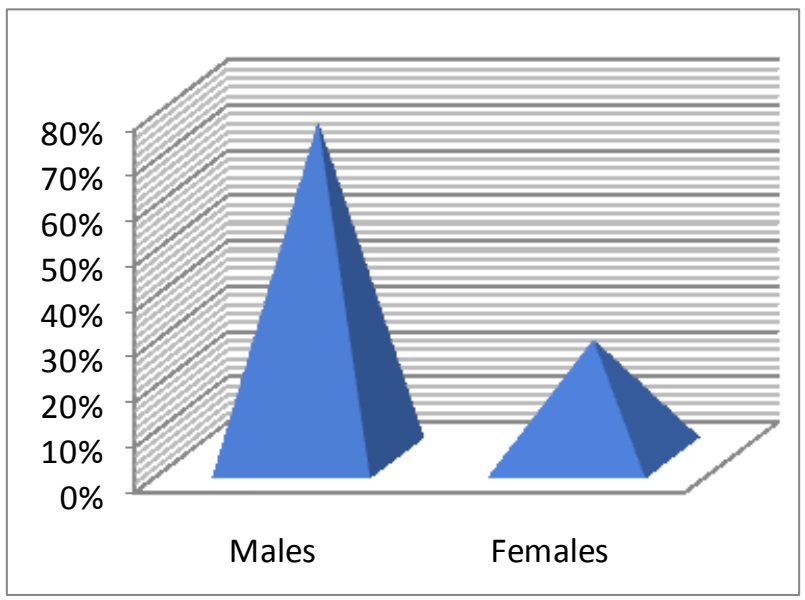

Figure 1. Gender distribution of hypertensive patients in chronic renal disease

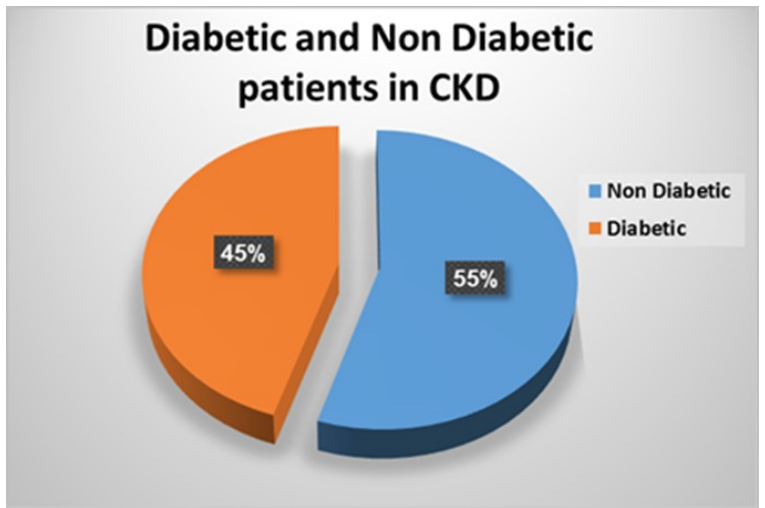

Figure 2: Pie chart showing percentage wise distribution of diabetic and nondiabetic in chronic renal disease patients

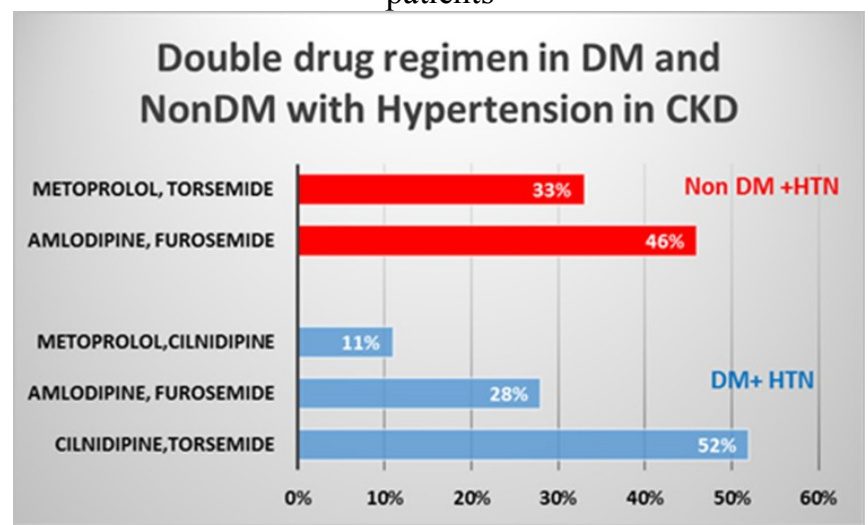

Figure 3: Bar chart showing double drug regimen in diabetic and nondiabetic with hypertension in chronic renal disease. 


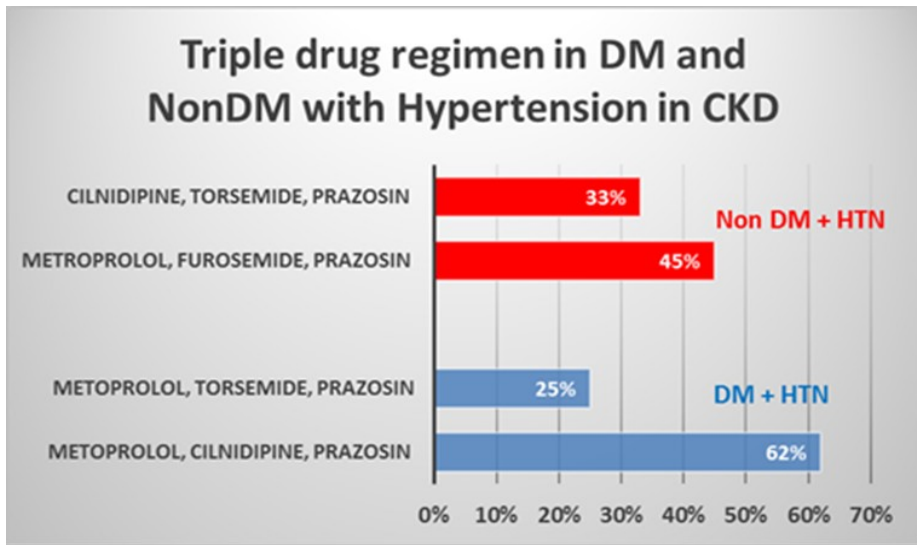

Figure 4: Bar chart showing triple drug regimen in diabetic and nondiabetic with hypertension in chronic renal disease.

\begin{tabular}{|l|l|}
\hline Characteristics & Number of patients ( $\mathbf{n}=\mathbf{1 2 0})$ \\
\hline Gender & $89(74 \%)$ \\
\hline Men & $31(26 \%)$ \\
\hline Women & $100(84 \%)$ \\
\hline Stages of CKD & $18(15 \%)$ \\
\hline Stage V [GFR $<15 \mathrm{~mL} / \mathrm{min} / 1.73 \mathrm{~m} 2]$ & $2(1.6 \%)$ \\
\hline Stage IV [ GFR $15-29 \mathrm{~mL} / \mathrm{min} / 1.73 \mathrm{~m} 2$ & \multicolumn{2}{|l|}{} \\
\hline Stage III [GFR $30-59 \mathrm{~mL} / \mathrm{min} / 1.73 \mathrm{~m} 2]$ & $120(100 \%)$ \\
\hline Comorbidities: & $54(45 \%)$ \\
\hline Hypertension & $51(43 \%)$ \\
\hline Diabetes & $13(11 \%)$ \\
\hline Anemia & $8(7 \%)$ \\
\hline Uremia & \\
\hline Coronary artery disease & \\
\hline CKD = Chronic kidney disease, GFR = Glomerular filtration rate & \\
\hline
\end{tabular}

Table1. Demographic characteristics of patients suffering from chronic kidney disease $(\mathrm{n}=120)$

\section{Discussion}

The Kidney Disease Outcomes Quality Initiative (K/DOQI) of the National Kidney Foundation (NKF) defines $\mathrm{CKD}$ as either kidney damage or a decreased glomerular filtration rate of less than 60 $\mathrm{mL} / \mathrm{min} / 1.73 \mathrm{~m}^{2}$ for 3 or more months ${ }^{[2]}$. Hypertension (HTN) has been reported to occur in $85 \%$ to $95 \%$ of patients with CKD (stages 3-5) ${ }^{[3]}$. The relationship between HTN and CKD is cyclic in nature. The progressive renal disease can exacerbate uncontrolled HTN due to volume expansion and increased systemic vascular resistance. Therefore, one of the main goals in CKD patients is to maintain blood pressure in the range recommended in different guidelines and to slow down the progression of renal disease and reduce cardiovascular morbidity and mortality ${ }^{[4-6]}$. In order to achieve and maintain adequate blood pressure control, most patients with CKD require multiple drugs combinations of antihypertensive agents ${ }^{[7]}$.

Angiotensin converting enzyme (ACE) Inhibitors or angiotensin receptor blockers (ARBs) studies have shown that these drugs target the renin-angiotensin system and prevent kidney function decline more so than other agents even achieving similar BP goals ${ }^{[8]}$. These results were found primarily in patients with proteinuria, whereas the benefit was less substantial for those without proteinuria. Based on these findings, guidelines recommend ACE inhibitor or ARB therapy as first-line treatment for those with diabetes or those presenting with nondiabetic kidney disease, HTN, and proteinuria. Treatment with both ACE inhibitor and ARB are not recommended, as these combinations has been shown to worsen kidney function. Combination of ACE inhibitor and ARB therapy did not reduce cardiovascular mortality or morbidity in comparison to monotherapy of an ACE inhibitor ${ }^{[9,10]}$. ACE inhibitors and ARBs are generally well tolerated. ACE inhibitors may cause a dry cough and angioedema are very rare.

Patients with CKD and HTN often experience fluid retention or fluid overload. As a result, diuretics are often necessary in their treatment regimen. Thiazides are recommended for patients with CKD stages 1 to 3 (GFR $\geq 30 \mathrm{~mL} / \mathrm{min}$ ) and have been established as effective agents for blood pressure and cardiovascular risk reduction. Loop diuretics are recommended for patients with CKD stage 4 to 5 (GFR $<30 \mathrm{~mL} / \mathrm{min}$ ) as they have been shown to be more effective in reducing extracellular fluid volume in patients with severely reduced GFR ${ }^{[2]}$. Thiazide diuretics (chlorthalidone, hydrochlorothiazide) and loop diuretics (bumetanide, furosemide, 
torsemide) all cause hyperuricemia (increased urination). Orthostatic hypotension may occur in response to any antihypertensive agents; however, it is common with diuretics.

Calcium channel blockers (CCBs) are considered second- or third-line therapy in the treatment of HTN in patients with $\mathrm{CKD}^{[11]}$. While there may be no difference in the effect on blood pressure lowering between non-dihydropyridine CCBs (ND-CCBs) e.g., diltiazem, verapamil) and dihydropyridine CCBs (e.g., amlodipine, nifedipine), ND-CCBs have been shown to significantly reduce proteinuria either when used alone or in combination with an ACE inhibitor or an $\mathrm{ARB}^{[12]}$. Common adverse effects include edema and constipation with ND-CCBs (especially verapamil) and flushing and peripheral edema with dihydropyridine agents ${ }^{[2]}$.

Aldosterone plays a severely deleterious role in the progression of CKD. Aldosterone receptor antagonists (e.g., spironolactone, eplerenone) may have a role of CKD therapy when blood pressure goals have not been achieved with first and second-line therapy. These agents have shown in human trials to provide a reduction in proteinuria when added to an $\mathrm{ACE}$ inhibitor or $\mathrm{ARB}{ }^{[13,14]}$. Beta-blockers can be considered as second- or third-line therapy if the patient has a compelling indication for a beta-blocker such as coronary artery disease or chronic heart failure ${ }^{[2]}$.

Out of the 120 case records evaluated, males predominated in the study population which is in agreement with the results of various other studies in India and United States ${ }^{[2]}$. The (mean $\pm \mathrm{SD}$ ) age of the patients was $56.5 \pm 13.2$ years with a range between 18 and 83 years. Average number of drugs per prescription was nine. The practice of polypharmacy is a common finding in similar studies in CKD patients with average number of drugs per prescription varying from 8 to $12.3^{[16,17]}$. In this study only the prescribed medicines were considered but it is well-known that over the counter use of medicines is common in India. This further increase the chances of drug interactions and adverse reactions. Combination of diuretic and calcium channel blockers ( $80 \%$ ) was commonly used in diabetic patient with hypertension. Cilnidipine was commonly used because of its longer duration of action of 24 hours, also prevents reflex tachycardia as it selectively acts on $\mathrm{N}$ type of calcium channels. Torsemide was preferred over furosemide because of longer duration of action and increased potency. Beta blocker because of its known adverse effects in diabetic patients with hypertension like negative effect on lipid profiles and hypoglycemic unawareness is getting less commonly used than in nondiabetic with hypertension. Use of antihypertensive drugs such as selective beta blocker, alpha-blocker, CCB combination (45 $\%)$ more commonly used in nondiabetic than in diabetics.

\section{Conclusion}

Our study identified a wide variety of drug classes were prescribed in a cohort of CKD patients indicative of prevailing morbidity. It provides a framework for continuous prescription audit in a hospital setting and suggests possible improvement in prescription practices in patients suffering from chronic kidney disease.

\section{Acknowledgements}

My sincere thanks to my husband Dr Rajkumar Yalawar in guiding and preparing the manuscript. Also thank my fellow colleagues, Dr Imran and Dr. Manjunath and dialysis unit staffs of Nephrology department.

Conflict of interest: Nil

Fund support: Nil:

\section{References}

[1] WORLD HEALTH ORGANIZATION: Burden of disease project. Available at http://www3.who.int/whosis/menu.cfm?

[2] National Kidney Foundation. KDOQI clinical practice guidelines for Chronic Kidney disease: Evaluation, classification, and stratification. Am J Kidney Dis 2002; 39:S1-266.

[3] Rao MV, Qiu Y, Wang C, Bakris G. Hypertension and CKD: Kidney Early Evaluation Program (KEEP) and National Health and Nutrition Examination Survey (NHANES), 1999-2004. Am J Kidney Dis. 2008; 51(suppl 2):S30-S37.

[4] Chobanian AV, Bakris GL, Black HR, et al. The seventh report of the Joint National Committee on Prevention, Detection, Evaluation, and Treatment of High Blood Pressure: the JNC 7 report. JAMA. 2003; 289:2560-2572.

[5] American Diabetes Association. Standards of medical care in diabetes-2012. Diabetes Care. 2012; 35(suppl 1):S1-S63.

[6] Bakris GL, Williams M, Dworkin L, et al. Preserving renal function in adults with hypertension and diabetes: a consensus approach. National Kidney Foundation Hypertension and Diabetes Executive Committees Working Group. Am J Kidney Dis. 2000; 36:64661 .

[7] Remuzzi G, Chiurchiu C, Ruggenenti P. Proteinuria predicting outcome in renal disease: nondiabetic nephropathies (REIN). Kidney Int Suppl. 2004; 66:S90-S96.

[8] Sharma P, Blackburn RC, Parke CL, et al. Angiotensin converting enzyme inhibitors and angiotensin receptor blockers for adults with early (stage 1 to 3 ) non-diabetic chronic kidney disease. Cochrane Database Syst Rev. 2011 ;( 10):CD007751.

[9] Van Vark LC, Bertrand M, Akkerhuis KM, et al. Angiotensin converting enzyme inhibitors reduce mortality in hypertension: a meta-analysis of randomized clinical trials of renin-angiotensin aldosterone system inhibitors involving 158,998 patients. Eur Heart J. 2012 Apr 17; Epub ahead of print.

[10] Chobanian AV, Bakris GL, Black HR, et al. The seventh report of the Joint National Committee on Prevention, Detection, Evaluation, and Treatment of High Blood Pressure: the JNC 7 report. JAMA. 2003; 289:2560-72.

[11] Bakris GL, Weir MR, Secic M, et al. Differential effects of calcium antagonist subclasses on markers of nephropathy progression. Kidney Int. 2004; 65:1991-2002. 
[12] Navaneethan SD, Nigwekar SU, Sehgal AR, et al. Aldosterone antagonists for preventing the progression of chronic kidney disease. Clin J Am Soc Nephrol. 2009; 4:542-551.

[13] Mehdi UF, Adams-Huet B, Raskin P, et al. Addition of angiotensin receptor blockade or mineralocorticoid antagonism to maximal angiotensin-converting enzyme inhibition in diabetic nephropathy. J Am Soc Nephrol. 2009; 20:2641-2650.

[14] Srishyla MV, Krishnamurthy M, Nagarani MA, Andrade C, Venketaraman BV.Prescription audit in an Indian hospital setting using the DD concept. Indian JPharmacol 1994; 26:23-8.

[15] Bailie GR, Eisele G, Liu L, Roys E, Kiser M, Finkelstein F, et al Patterns of medication use in the RRI-CKD study: Focus on medication with cardiovascular effect. Nephrol Dial Transplant 2005; 20:1110-5.

[16] Manley HJ, Garvin CG, Drayer DK, Reid GM, Bender WL, Neufeld TK, et al. Medication prescription pattern in ambulatory hemodialysis patients: Comparison of USRDS to a large not for profit dialysis provider. Nephrol Dial Transplant1 2004; 19:1842-8.

[17] Junius-Walker U, Theile G, Hummers-Pradier E. Prevalence and predictors of polypharmacy among older primary care patients in Germany. FAM Pract 2007; 24:14-9. 